\title{
Inhibition of Shigella sonnei-induced epithelial barrier disruption by surface- layer associated proteins of lactobacilli from Chinese fermented food
}

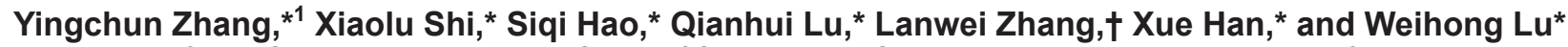 \\ *Department of Food Science and Engineering, School of Chemistry and Chemical Engineering, Harbin Institute of Technology, \\ Harbin 150001, China \\ †College of Food Science and Engineering, Ocean University of China, Qingdao 266003, China
}

\begin{abstract}
Surface-layer associated proteins (SLAP) of Lactobacillus paracasei ssp. paracasei M5-L and Lactobacillus casei Q8-L were examined to identify the functional basis for their protection within intestinal epithelial cells. The results showed that SLAP of M5-L and Q8-L remained active in a trypsin solution and retained a 45$\mathrm{kDa}$ protein band, similar to that observed in controls. In contrast, under conditions of simulated gastric juice, the SLAP were partially degraded. Inhibitory effects of SLAP on adherence of Shigella sonnei to HT-29 cells were assessed with use of exclusion, competition, and replacement assays. In response to M5-L at $50 \mu \mathrm{g} / \mathrm{mL}$ SLAP, an inhibition ratio of $33 \%$ was obtained, while for Q8-L at $400 \mu \mathrm{g} / \mathrm{mL}$ SLAP, the inhibition ratio was $48 \%$. Hoechst 33258 test results showed that cells infected with $S$. sonnei and co-incubated with SLAP of M5-L and Q8-L were only partially apoptotic, with apoptosis rates of 37.67 and $43.67 \%$, respectively. These levels of apoptosis were substantially lower than that observed with cells infected with $S$. sonnei alone. In addition, the SLAP of Q8-L and M5-L reduced downstream caspase-1 activity and further modified apoptotic cell damage. Finally, SLAP of M5-L and Q8-L were also able to prevent $S$. sonnei-induced membrane damage by inhibiting delocalization of zonula occludens (ZO)-1 and reducing the amount of occludin produced by $S$. sonnei.
\end{abstract}

Key words: surface-layer associated proteins, lactobacilli, inhibition, Shigella sonnei

\section{INTRODUCTION}

Shigella, a pathovar of Escherichia coli, is composed of 4 distinct species: Shigella flexneri, Shigella sonnei, Shigella dysenteriae, and Shigella boydii. Shigella accounts for the majority of dysentery invading the human gut

Received June 29, 2017.

Accepted November 22, 2017.

${ }^{1}$ Corresponding author: zyc229@163.com mucosa and is the basis for most gastrointestinal illness infections occurring worldwide (Anderson et al., 2016). Direct contact with and invasion of host epithelial and immune cells are the main modes of host-pathogen interactions, a process that is particularly characteristic of Shigella (Tran Van Nhieu et al., 2000). Pathogenic bacteria can regulate tight junction (TJ) structures of epithelial cells, and the role of TJ in infection has been widely demonstrated. Specifically, disruption of TJ leads to a distinct increase in paracellular permeability and polarity defects, which then facilitate viral or bacterial entry and subsequent dispersal. In addition to pathological changes in TJ integrity, TJ proteins such as occludin and claudins can either function as receptors for pathogen entry or interact with viral or bacterial effector molecules, which represents an essential step for infection (Lu et al., 2014).

Many lactobacilli have been characterized as probiotics because they possess the ability to exert health benefits for the host. The activities of probiotics have been investigated and several modes of action proposed, including co-aggregation with pathogens, competitive exclusion, contribution to mucosal barrier function, antimicrobial activity through reductions in luminal $\mathrm{pH}$, secretion of specific compounds such as bacteriocins, and the modulation of immune responses (Golowczyc et al., 2007; Breuer, 2009; Šušković et al., 2010; Bron et al., 2011). Adhesion to intestinal epithelial cells is considered the first step for the probiotic function of lactobacilli. Lactobacilli with high adhesive abilities have a greater gut residence time, increase the exclusion of pathogens, and protect epithelial cells (Servin, 2004; Granato et al., 1999). Accordingly, the survival and persistence of lactobacilli within the gastrointestinal tract represents an important factor with regard to their beneficial effects (Zhang et al., 2013).

Many Lactobacillus species possess surface-layer associated proteins (SLAP), which are surface structures consisting of 2-dimensional arrays of proteinaceous subunits that are found among the most commonly observed prokaryotic cells (Sleytr, 1976; Sleytr et al., 2014). Several functions of SLAP have been reported, 
such as protection against hostile environments, maintenance of cellular shape, control of nutrient and metabolite transfer, and they play an important role in the colonization of Lactobacillus acidophilus within the gastrointestinal tract (GIT; Beveridge et al., 1997; Chen et al., 2007). The SLAP are also involved in mediating adhesion of lactobacilli cells to intestinal epithelium cells (Khang et al., 2009; Sun et al., 2013), as well as inhibiting Salmonella-induced reorganization of the cytoskeleton (Li et al., 2012). The adhesive quality of intestinal epithelia allows lactobacilli to prolong their probiotic effects (Avall-jääskeläinen et al., 2003; Jakava-Viljanen and Palva, 2007).

The isolates Lactobacillus paracasei ssp. paracasei M5-L and Lactobacillus casei Q8-L, which can survive passage through the GIT and exert antimicrobial activity against 3 pathogens (Shigella sonnei ATCC 25931, Escherichia coli ATCC25922, and Salmonella enterica serovar Typhimurium ATCC 14028), have been demonstrated to adhere effectively to HT-29 cells (Zhang et al., 2011). In addition, M5-L and Q8-L possess the ability to inhibit adherence of $S$. sonnei to HT-29 cells (Zhang et al., 2010). The SLAP of M5-L and Q8-L have been identified as surface antigens and the elongation factor $\mathrm{Tu}$ (GI number: 489671527), respectively, as determined by liquid chromatography-tandem MS (Zhang et al., 2016).

The main objective of this study was to evaluate the inhibitory effects of SLAP on adherence of S. sonnei to HT-29 cells by using exclusion, competition, and replacement assays. In addition, we evaluated the protective ability of SLAP on intestinal epithelial cells to verify that M5-L and Q8-L could serve as novel probiotic strains for use in the food industry. The results of this study could help to identify probiotic candidates for further investigation in vivo.

\section{MATERIALS AND METHODS}

\section{Bacterial Strains and Growth Conditions}

Lactobacillus paracasei ssp. paracasei M5-L was isolated from kumiss produced in local households in Sinkiang (China), whereas L. casei Q8-L was isolated from yak yogurt produced in local households in Qinghai, China (Zhang et al., 2011). Both strains were stored in our laboratory and cultured in de Man, Rogosa, and Sharpe (Difco/Becton Dickinson Co., Franklin Lakes, NJ) broth under aerobic condition at $37^{\circ} \mathrm{C}$. The indicator bacteria used for antimicrobial assays were cultured in tryptone soy agar (TSA) or broth in aerobic conditions at $37^{\circ} \mathrm{C}$ and included S. sonnei ATCC 25931 provided by the Microbiological Laboratory of the Clinical Detection Center of Heilongjiang (Harbin, China).

\section{Extraction of SLAP}

The SLAP of L. paracasei ssp. paracasei M5-L and $L$. casei Q8-L were extracted with $5 \mathrm{M} \mathrm{LiCl}$ as described previously (Lortal et al., 1992; Smit et al., 2001). Briefly, cells obtained from $500 \mathrm{~mL}$ of de Man, Rogosa, and Sharpe broth after $16 \mathrm{~h}$ of incubation were collected by centrifugation $\left(8,000 \times g\right.$ at $10^{\circ} \mathrm{C}$ for $\left.10 \mathrm{~min}\right)$, washed twice with ice-cold sterile distilled water (overnight at $4^{\circ} \mathrm{C}$ ), and then mixed with $50 \mathrm{~mL}$ of $5 \mathrm{M} \mathrm{LiCl}$. The mixture was incubated in a shaking incubator (SPX$150 \mathrm{C}$, Boxun, China) at $150 \mathrm{rpm}$ and $37^{\circ} \mathrm{C}$ for $60 \mathrm{~min}$ to extract noncovalently bound proteins. The supernatant was then harvested by centrifugation $(10,000 \times g$ at $10^{\circ} \mathrm{C}$ for $20 \mathrm{~min}$ ) and dialyzed extensively against distilled water, with the water being changed every $2 \mathrm{~h}$ for the first $8 \mathrm{~h}$. Then, the $\mathrm{LiCl}$ was removed from the liquid, concentrated with PEG-8000 (polyethylene glycol, Amresco, Solon, $\mathrm{OH}$ ), and stored at $-20^{\circ} \mathrm{C}$ before being lyophilized.

\section{SLAP of Lactobacilli Activity Under Conditions Simulating the Human GIT}

Resistance of the SLAP of M5-L and Q8-L to pepsin under low $\mathrm{pH}$ was tested as described previously (Charteris et al., 1998). Briefly, simulated gastric juice was prepared by suspending pepsin $(0.3 \mathrm{mg} / \mathrm{mL}$; Sigma, St. Louis, MO) in PBS (pH 2.0). Subsequently, $0.5 \mathrm{~mL}$ of SLAP of M5-L or Q8-L were inoculated into $1 \mathrm{~mL}$ of this simulated gastric juice and $0.3 \mathrm{~mL}$ of $\mathrm{NaCl}(0.5 \%$ wt/vol), mixed, and incubated at $37^{\circ} \mathrm{C}$ for $1 \mathrm{~h}$. The degradation of SLAP was analyzed using SDS-PAGE. With this protocol, the time spent by food in the stomach could be modeled.

Tolerance of conditions of the small intestine was tested in PBS ( $\mathrm{pH} 8.0)$ containing trypsin $(0.1 \mathrm{mg} / \mathrm{mL}$, Sigma) and $0.3 \%$ (wt/vol) Oxgall (Sigma). The SLAP $(0.5 \mathrm{~mL})$ of $\mathrm{M} 5-\mathrm{L}$ and $\mathrm{Q} 8-\mathrm{L}$ were inoculated into $1 \mathrm{~mL}$ of the simulated small intestine juice, mixed, and incubated at $37^{\circ} \mathrm{C}$ for $3 \mathrm{~h}$. The degradation of SLAP was analyzed using SDS-PAGE, to test transit tolerance within this simulated small intestine, again providing a model for the time spent by food in the small intestine (Maragkoudakis et al., 2006).

\section{Antimicrobial Activity of SLAP}

Cells of $S$. sonnei from $1 \mathrm{~mL}$ of TSA culture medium after $12 \mathrm{~h}$ of incubation were collected by centrifugation $\left(8,000 \times g\right.$ at $10^{\circ} \mathrm{C}$ for $\left.10 \mathrm{~min}\right)$. These cells were then inoculated at varying concentrations with $100 \mu \mathrm{L}$ of $\operatorname{SLAP}(0,25,50$, or $100 \mu \mathrm{g} / \mathrm{mL})$ in RPMI-1640 medium (the cell culture medium was added to the $1 \mathrm{~mL}$ of 
TSA) and incubated for $2 \mathrm{~h}$ at $37^{\circ} \mathrm{C}$ in $5 \% \mathrm{CO}_{2}$. Shigella sonnei cells were then serially diluted and plated on TSA plates to determine their survival. All experiments were independently performed 3 times and each assay was replicated 3 times.

\section{Inhibition by SLAP of S. sonnei Adherence to HT-29 Cells}

The ability for SLAP to inhibit $S$. sonnei adherence to HT-29 cells was assayed according to methods reported previously with some modifications (Candela et al., 2008; Ramiah et al., 2008). In brief, approximately 3 to $4 \times 10^{5}$ cells per well were seeded in a 12 -well plate (Corning Inc., Corning, NY). The culture medium was changed daily and HT-29 monolayers at 80 to $90 \%$ confluence were washed twice with PBS ( $\mathrm{pH} 7.4)$ before experiments.

In the exclusion assay, HT-29 monolayers were inoculated with $800 \mu \mathrm{L}$ of RPMI-1640 medium; then, varying concentrations of $100 \mu \mathrm{L}$ of $\operatorname{SLAP}(25,50$, 100 , or $400 \mu \mathrm{g} / \mathrm{mL}$ ) were added to the HT-29 cells and incubated for $60 \mathrm{~min}$ at $37^{\circ} \mathrm{C}$ in $5 \% \mathrm{CO}_{2}$, followed by atmospheric incubation for $1 \mathrm{~h}$ at $37^{\circ} \mathrm{C}$ in $5 \% \mathrm{CO}_{2}$. The HT-29 monolayers were then inoculated with $100 \mu \mathrm{L}$ of $S$. sonnei suspension $\left(10^{7} \mathrm{cfu} /\right.$ well $)$ in RPMI- 1640 medium and incubated for $1 \mathrm{~h}$ at $37^{\circ} \mathrm{C}$ in $5 \% \mathrm{CO}_{2}$.

In the competition assay, HT-29 monolayers were inoculated with $800 \mu \mathrm{L}$ of RPMI-1640 medium, and a mixture of varying concentrations of $100 \mu \mathrm{L}$ of SLAP $(25,50,100$, or $400 \mu \mathrm{g} / \mathrm{mL})$ and $100 \mu \mathrm{L}$ of $S$. sonnei suspension ( $10^{7} \mathrm{cfu} /$ well) in RPMI-1640 medium were added into each well and cultured for $2 \mathrm{~h}$ at $37^{\circ} \mathrm{C}$.

In the displacement assay, HT-29 monolayers were inoculated with $800 \mu \mathrm{L}$ of RPMI-1640 medium and 100 $\mu \mathrm{L}$ of $S$. sonnei suspension $\left(10^{7} \mathrm{cfu} /\right.$ well $)$ in RPMI-1640 medium and incubated for $1 \mathrm{~h}$ at $37^{\circ} \mathrm{C}$ in $5 \% \mathrm{CO}_{2}$. Nonadhering cells were removed by washing twice with sterile PBS. The HT-29 monolayers were then inoculated with varying concentrations of $\operatorname{SLAP}(25,50,100$, or $400 \mu \mathrm{g} / \mathrm{mL}$ ) in RPMI-1640 medium and incubated for $1 \mathrm{~h}$ at $37^{\circ} \mathrm{C}$ in $5 \% \mathrm{CO}_{2}$. Wells containing $S$. sonnei alone served as controls.

In all experiments, nonadhering cells were removed by washing 4 times with sterile PBS, and then treated with $0.5 \mathrm{~mL}$ of $0.5 \%$ (vol/vol) Triton X-100 (Amresco) for $5 \mathrm{~min}$ in an ice water bath. The cells were then serially diluted and plated onto TSA plates to count $S$. sonnei cells. The ability of SLAP to exclude, compete with, and displace $S$. sonnei was determined by comparing the adhesion of $S$. sonnei in the presence of SLAP with that of $S$. sonnei alone. The number of $S$. sonnei cells failing to adhere to HT-29 cells was then expressed as a percentage. All experiments were replicated 3 times and each assay was performed in triplicate.

\section{Effects of SLAP on Apoptosis of Human Cells Using Hoechst 33258 Staining}

Cell apoptosis was detected by nucleus staining with Hoechst 33258 as described previously (Allen et al., 2001). Briefly, $2 \mathrm{~mL}$ of HT-29 cells $\left(2.5 \times 10^{5}\right.$ cells/ well) was cultured in a 6 -well plate at $37^{\circ} \mathrm{C}$ for $6 \mathrm{~h}$ after cells had adhered to the wells, followed by addition of $200 \mu \mathrm{L}$ of SLAP of M5-L $(50 \mu \mathrm{g} / \mathrm{mL})$ or Q8-L $(400 \mu \mathrm{g} /$ $\mathrm{mL})$ and $200 \mu \mathrm{L}$ of $S$. sonnei $\left(1 \times 10^{7} \mathrm{cfu} / \mathrm{mL}\right)$ to the HT-29 cells. Medium (RPMI-1640) was added to the well to achieve a final volume of $2 \mathrm{~mL}$ and co-incubated for $12 \mathrm{~h}$ at $37^{\circ} \mathrm{C}$ in $5 \% \mathrm{CO}_{2}$. Treated and control cells were washed twice with PBS and stained using Hoechst $33258(5 \mu \mathrm{g} / \mathrm{mL}$ from a stock solution of $1 \mathrm{mg} / \mathrm{mL}$ in sterile water) for $5 \mathrm{~min}$ at $37^{\circ} \mathrm{C}$. The stained cells were manually shaken several times. The dye liquor was aspirated and cells were washed twice with PBS and observed immediately under a fluorescence microscope (Olympus/Nikon, Tokyo, Japan) with a filter set (excitation/emission) of BP330-380 nm/LP420 nm. Images were recorded with use of a color charge-coupled device camera.

\section{Caspase-1 Activity Assays}

Caspase-1 activity was determined using a kit (Beyotime Institute of Biotechnology, Jiangsu, China), based on the ability of caspase-1 to change acetyl-Tyr-ValAla-Asp $p$-nitroanilide (Ac-YVAD- $p \mathrm{NA}$ ) into yellow formazan $p$-nitroaniline. Cell lysates were centrifuged at $12,000 \times g$ for $10 \mathrm{~min}$, and the protein concentrations were determined by the Bradford protein assay. Cellular extracts were incubated in a 96-well microtiter plate with $20 \mathrm{ng}$ of caspase substrate acetyl-Asp-GluVal-Asp $p$ NA (Ac-DEVD- $p$ NA) overnight at $37^{\circ} \mathrm{C}$. The absorbance values of $p \mathrm{NA}$ at $405 \mathrm{~nm}$ (optical density at $405 \mathrm{~nm}, \mathrm{OD}_{405}$ ) were measured using a 96-well plate reader (BioTek, Santa Barbara, CA). An increase in $\mathrm{OD}_{405}$ indicated activation of caspase-1 (Singh et al., 2001).

\section{Immunochemistry Analysis of Distribution of Zonula Occludens-1 and Occludin}

The distribution of zonula occludens-1 (ZO-1) and occludin were analyzed following infection (treatment of HT-29 cells according to the methods described in the subsection Effects of SLAP on Apoptosis of $\mathrm{Hu}-$ man Cells Using Hoechst 33258 Staining) using im- 
munochemistry and confocal microscopy. Monolayers were washed extensively with PBS and fixed with $2 \%$ of paraformaldehyde for $30 \mathrm{~min}$ at $37^{\circ} \mathrm{C}$. The cells were permeabilized with $0.1 \%$ Triton-X100 in PBS for 5 min at $37^{\circ} \mathrm{C}$ and washed with PBS. Polyclonal rabbit antiZO-1 antibody (bs-1329R, Boaoseng, Beijing, China) and polyclonal rabbit anti-occludin antibody (bs-1495R, Boaoseng) were diluted in PBS and incubated with permeabilized cells for $45 \mathrm{~min}$ at room temperature in a humid atmosphere. The monolayers were washed and treated with fluorescein-conjugated anti-rabbit IgG (SV-0002, Boaoseng) for $45 \mathrm{~min}$ at room temperature in a humid atmosphere. After washing with PBS, the filters were excised from their supports, mounted, and observed under a Leica confocal laser scanning microscope (Leica, Shanghai, China).

\section{Statistical Analysis}

All experiments were performed 3 times independently and each assay was performed in duplicate. Results were expressed as means \pm standard deviation. The level of significance was analyzed by ANOVA $(P<$ 0.05) using SPSS14.0 for Windows (evaluation version; SPSS Inc., Chicago, IL).

\section{RESULTS}

\section{Tolerance of SLAP Under Conditions Simulating the Human GIT}

Under conditions of simulating gastric juice, the SLAP of M5-L were degraded to between 35 and 25 $\mathrm{kDa}$ (lane 4) compared with controls (lane 2), as shown in Figure 1. Results obtained from SDS-PAGE showed that SLAP of M5-L were degraded to low-molecularweight proteins. The SLAP of Q8-L were not degraded and the main band of Q8-L SLAP was at $45 \mathrm{kDa}$ (Figure 1; lane 6), which was similar to that of controls (Figure 1; lane 7). These findings indicate that the SLAP of Q8-L demonstrated strong tolerance to degradation after incubation with simulated gastric juice for $1 \mathrm{~h}$ (Figure 1). No protein band associated with pepsin was displayed as shown in Figure 1.

In contrast to results obtained under conditions of simulated gastric juice, the SLAP of M5-L and Q8-L remained active in trypsin solution at $\mathrm{pH} 8.0$ and in the presence of bile salts (Oxgall, $0.3 \% \mathrm{wt} / \mathrm{vol}$ ). Even after $4 \mathrm{~h}$ of exposure, they retained 45 -kDa protein bands, as shown in Figure 1 (lanes 3 and 5, respectively), which was similar to that observed in controls (lanes 2 and 7 , respectively). No protein band associated with trypsin was displayed as shown in Figure 1 (lane 8).

\section{Antimicrobial Activity of SLAP}

Neither SLAP of M5-L nor Q8-L showed any significant antimicrobial activity compared with the control group $(P>0.05)$. The viability of $S$. sonnei did not decrease and different concentrations of SLAP did not affect the survival of $S$. sonnei (Table 1). Based upon these results, we can exclude the possibility that any antimicrobial activity of the tested SLAP was exerted upon S. sonnei.

\section{SLAP Inhibition of S. sonnei Adherence to HT-29 Cells}

At different concentrations, SLAP of M5-L showed varying effects on $S$. sonnei adherence to HT-29 cells in competition, exclusion, and displacement assays, as summarized in Figure 2A. The SLAP of M5-L were more effective in their capacity to inhibit $S$. sonnei adherence to HT-29 cells in competition and exclusion assays than in the displacement assay. Overall, $50 \mu \mathrm{g} / \mathrm{mL}$ SLAP showed maximal inhibition rates on $S$. sonnei with adherence prevented at $45.49,48.12$, and $33.08 \%$ [\% S. sonnei prevented from adherence to HT-29 cells $\%$ (control - test)/control] in competition, exclusion, and displacement assays, respectively $(P<0.05)$. Data are means \pm standard deviation of 3 independent experiments; S. sonnei alone served as a control

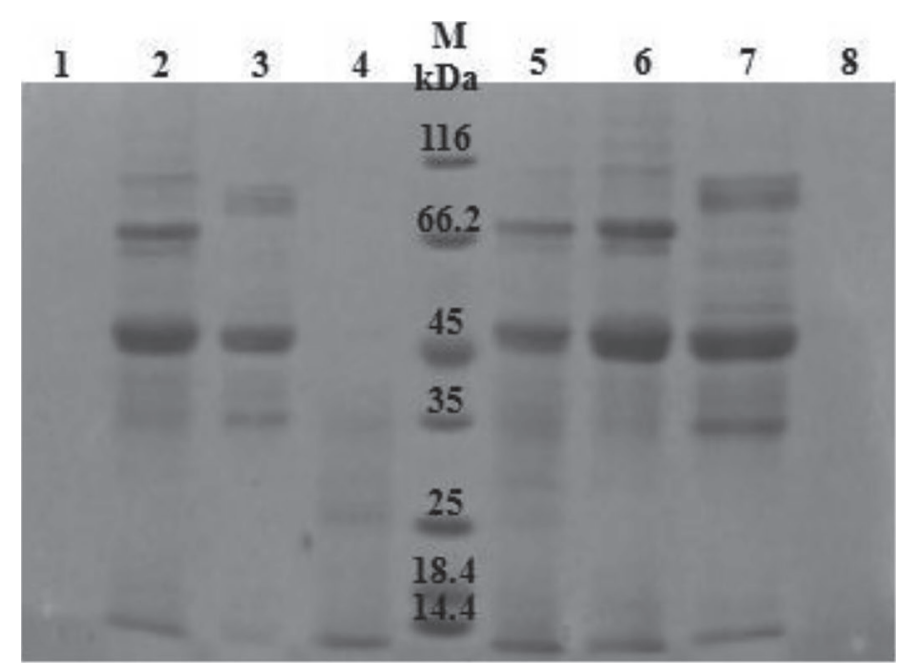

Figure 1. Sodium dodecyl sulfate-PAGE of surface-layer proteins under in vitro conditions simulating gastric and intestinal juice. $\mathrm{M}=$ low-molecular-weight protein standards; lane $1=$ trypsin alone; lane $2=$ surface-layer associated protein (SLAP) of Lactobacillus paracasei ssp. paracasei M5-L; lane $3=$ SLAP of M5-L treated with trypsin; lane $4=\mathrm{SLAP}$ of M5-L treated with pepsin; lane $5=\mathrm{SLAP}$ of Lactobacillus casei Q8-L treated with trypsin; lane $6=$ SLAP of Q8-L treated with pepsin (lane 6 ); lane $7=\mathrm{SLAP}$ of Q8-L; and lane $8=$ pepsin alone. 
Table 1. Antimicrobial activity of surface-layer associated proteins (SLAP) of Lactobacillus paracasei ssp. paracasei M5-L and Lactobacillus casei Q8-L on Shigella sonnei $(0 \mu \mathrm{g} / \mathrm{mL}$ SLAP of M5-L or Q8-L was the baseline level for $S$. sonnei $)^{1}$

\begin{tabular}{ll}
\hline SLAP & $\begin{array}{l}\text { Shigella sonnei } \\
\left(\times 10^{9} \mathrm{cfu} / \mathrm{mL}\right)\end{array}$ \\
\hline SLAP of M5-L $(\mu \mathrm{g} / \mathrm{mL})$ & \\
0 & $1.93 \pm 0.10^{\mathrm{a}}$ \\
25 & $2.28 \pm 0.16^{\mathrm{a}}$ \\
50 & $2.05 \pm 0.04^{\mathrm{a}}$ \\
100 & $2.00 \pm 0.04^{\mathrm{a}}$ \\
& \\
SLAP of Q8-L $(\mu \mathrm{g} / \mathrm{mL})$ & \\
0 & $1.84 \pm 0.06^{\mathrm{a}}$ \\
25 & $1.85 \pm 0.17^{\mathrm{a}}$ \\
50 & $1.99 \pm 0.06^{\mathrm{a}}$ \\
100 & $1.82 \pm 0.16^{\mathrm{a}}$ \\
\hline
\end{tabular}

${ }^{a}$ Means with the same superscript letter do not differ significantly $(P$ $<0.05)$.

${ }^{1}$ Data are means $\pm \mathrm{SD}$ of 3 independent experiments; $0 \mu \mathrm{g} / \mathrm{mL}$ of SLAP alone served as a control.

The most effective inhibition rates in response to varying concentrations of SLAP of Q8-L on S. sonnei were obtained in the exclusion assay. The SLAP of Q8-L at $400 \mu \mathrm{g} / \mathrm{mL}$ significantly $(P<0.05)$ inhibited $S$. sonnei and adherence of $S$. sonnei to HT-29 cells was inhibited $37.84,40.60$, and $40.60 \%$ in the exclusion, competition, and displacement assays, respectively $(P$ $<0.05$; Figure 2B).

\section{Effects of SLAP on S. sonnei-Induced Human Cell Apoptosis}

Under fluorescence microscopy, normal cells showed a uniform dispersion of circular fluorescence and displayed many villus projections on their surface and an even distribution of nuclear chromatin. In contrast, typical apoptotic cells had densely stained nuclei with a half-moon fluorescence shape located in the nucleus and cytoplasm, irregular nuclear shapes, and condensation and fragmentation of nuclear chromatin.

The results of cellular apoptotic rates are summarized in Table 2. Cells treated with $S$. sonnei alone were apoptotic in all sections sampled, with an apoptosis rate of $100 \%$ (Figure 3D). Cells treated with SLAP of M5-L and Q8-L alone were essentially normal, with apoptosis rates of only 5\% (Figure 3B) and 10.67\% (Figure 3C), respectively. These results indicate that SLAP exerted no effects on the normal growth processes of these cells. Cells infected with $S$. sonnei and co-incubated with SLAP of M5-L or Q8-L were only partially apoptotic with apoptosis rates of $37.67 \%$ (Figure $3 \mathrm{E}$ ) and $43.67 \%$ (Figure $3 \mathrm{~F}$ ), respectively. These rates were lower than that of apoptosis rates of cells with $S$. sonnei alone, indicating that SLAP exerted a protective effect on cells invaded by $S$. sonnei.

\section{Caspase-1 Activity Assays}

Caspases are a family of cysteine-aspartic proteases that modulate apoptotic responses. In the absence of apoptotic stimuli, caspases exist in an inactive form as procaspases (Riedl and Salvesen, 2007). Therefore, evaluation of initiator caspase activation can be used to determine the apoptotic pathways involved. To assess the effects of SLAP of M5-L and Q8-L on caspase-1 activation after infection of intestinal epithelial cells with $S$. sonnei, we determined caspase-1 activity changes after infection. The SLAP of M5-L (Figure 4A) significantly inhibited caspase-1 activity as demonstrated
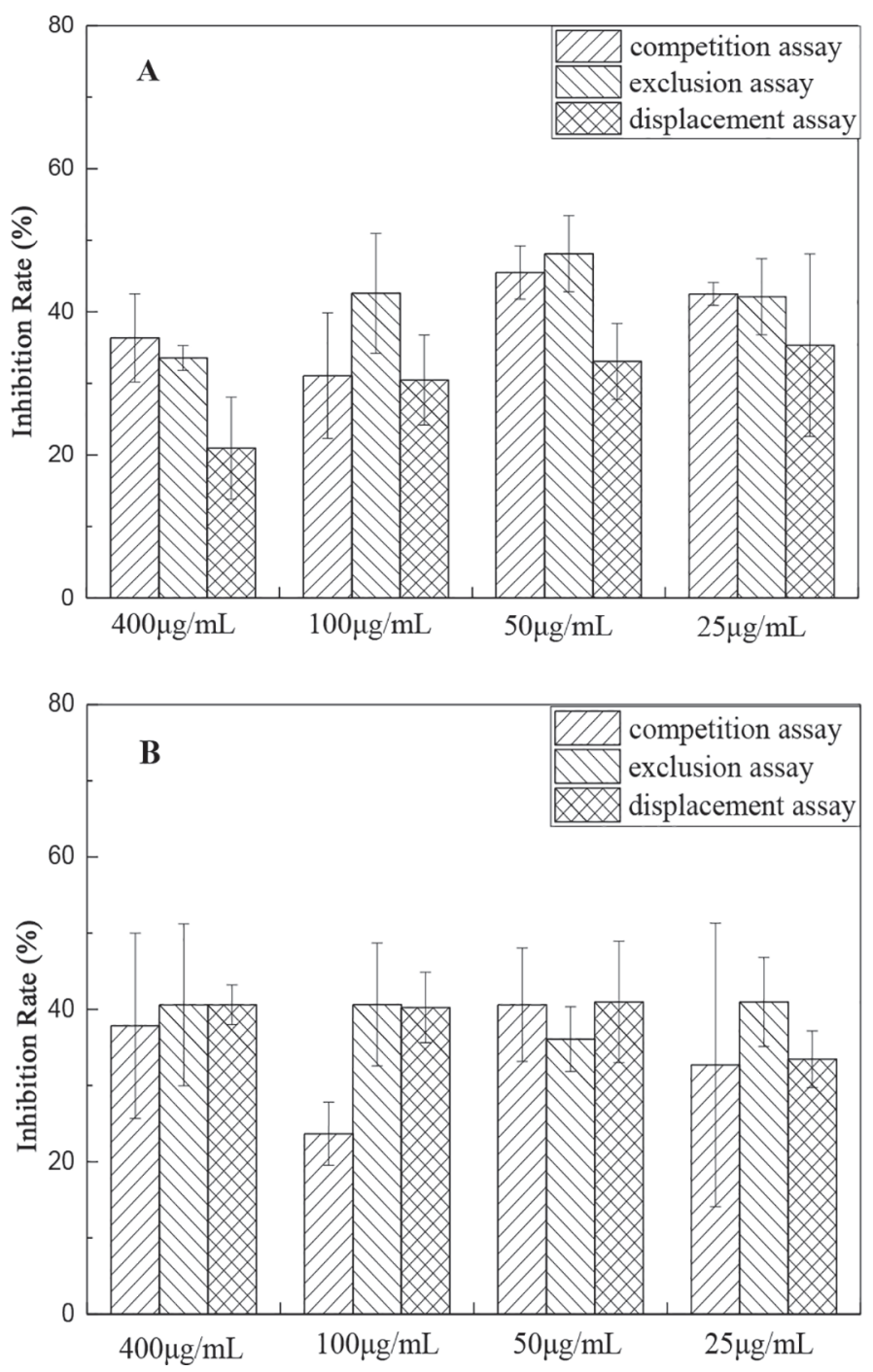

Figure 2. Inhibition rates resulting from varying concentrations of surface-layer associated proteins (SLAP) of Lactobacillus paracasei ssp. paracasei M5-L (A) and Lactobacillus casei Q8-L (B) on Shigella sonnei. Data are means \pm SD of 3 independent experiments; S. sonnei alone served as a control $[\%$ S. sonnei prevented from adherence to HT- 29 cells \% (control - test)/control]. 

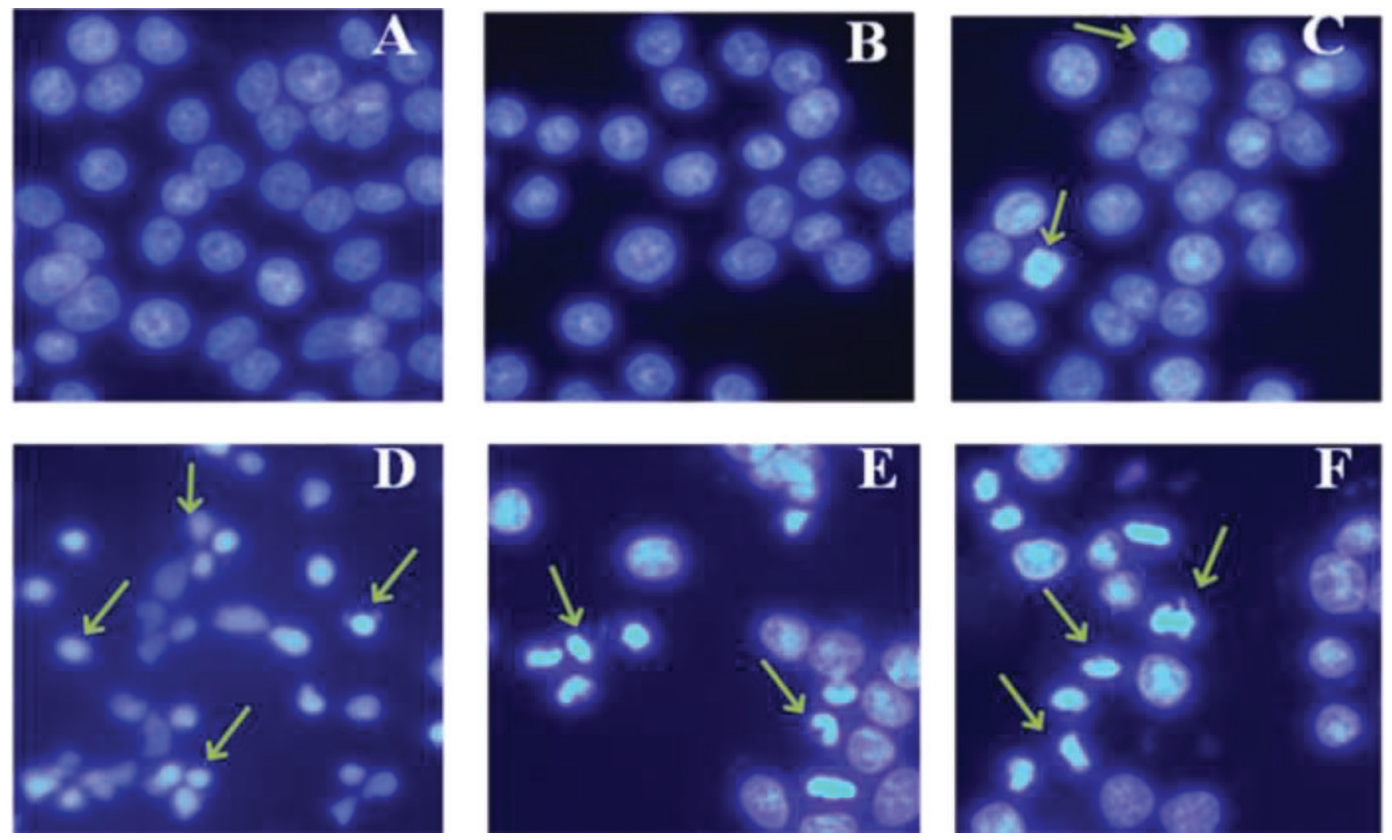

Figure 3. The surface-layer associated proteins (SLAP) of lactobacilli inhibited Shigella sonnei-induced human cell apoptosis. (A) Intact monolayer of uninfected HT-29 cells; (B) treatment of HT-29 cells with SLAP of Lactobacillus paracasei ssp. paracasei M5-L alone; (C) treatment of HT-29 cells with SLAP of Lactobacillus casei Q8-L alone; (D) treatment of HT-29 cells with S. sonnei alone; (E) HT-29 cells infected with S. sonnei and co-incubated with SLAP of M5-L; and (F) HT-29 cells infected with S. sonnei and co-incubated with SLAP of Q8-L. Arrows indicate cell apoptosis. Color version available online.

by the exclusion (17.81 $\pm 0.26 \%$ reduction) and competition (30.22 $\pm 0.32 \%$ reduction) assays compared with that observed in response to $S$. sonnei alone $(P$ $<0.05)$. No decrease in caspase-1 activity by SLAP of M5-L was observed in the displacement assay compared with $S$. sonnei alone $(P>0.05)$. However, compared with $S$. sonnei alone, SLAP of Q8-L (Figure 4B) significantly inhibited caspase- 1 activity in all 3 assays, with reductions of $42.19 \pm 0.28 \%, 30.87 \pm 0.73 \%$, and 22.63 $\pm 0.27 \%$ observed in the exclusion, competition, and displacement assays, respectively $(P<0.05)$. These

Table 2. Apoptosis of HT-29 cells determined by Hoechst 33258 fluorescent analysis after co-incubation with Shigella sonnei and Lactobacillus SLAP (M5-L and Q8-L), which are illustrated in Figure 3

\begin{tabular}{lc} 
Treatment $^{1}$ & $\begin{array}{c}\text { Apoptosis rate of } \\
\text { HT-29 } \text { cells }^{2}(\%)\end{array}$ \\
\hline Control (uninfected HT-29 cells) & 0 \\
SLAP of M5-L & $5 \pm 2.65$ \\
SLAP of Q8-L & $10.67 \pm 2.52$ \\
Shigella sonnei & 100 \\
SLAP of M5-L + S. sonnei & $37.67 \pm 3.52$ \\
SLAP of Q8-L + S. sonnei & $43.67 \pm 3.51$ \\
\hline
\end{tabular}

${ }^{1} \mathrm{SLAP}=$ surface-layer associated protein; $\mathrm{M} 5-\mathrm{L}=$ Lactobacillus paracasei $\mathrm{ssp}$. paracasei $\mathrm{M} 5-\mathrm{L}$; Q8-L = Lactobacillus casei $\mathrm{Q} 8-\mathrm{L}$.

${ }^{2}$ Data are means $\pm \mathrm{SD}$ of 3 independent experiments. findings demonstrate that SLAP of M5-L and Q8-L can reduce $S$. sonnei-induced apoptosis in HT-29 cells by inhibiting the activation of caspase- 1 as revealed in all 3 assays for Q8-L and in the exclusion and competition assays for M5-L.

\section{Distribution of ZO-1 and Occludin}

Basal concentrations of ZO-1 and occludin antibodies were determined in a pretrial experiment by immunohistochemical analysis. The observation of brown portions distributed uniformly along the cell membrane indicated that optimal concentrations of ZO-1 and occludin antibodies were obtained with a 1:100 dilution. The results of this experiment revealed that the ZO-1 protein was visible in the cytoplasm and occludin was distributed along the outer membrane, which verified that the ZO-1 protein was located in the cell membrane and occludin located extracellularly (data not shown).

The SLAP of M5-L and Q8-L significantly increased the production of ZO-1 and occludin, whereas S. sonnei decreased production of ZO-1 compared with that of the control group. When cells infected with $S$. sonnei were co-incubated with the SLAP of M5-L and Q8-L, S. sonnei-induced ZO-1 and occludin distributions were inhibited compared with $S$. sonnei-infected cells alone (Figure 5). 


\section{DISCUSSION}

The in vitro testing of lactobacilli-induced SLAP activity in simulated GIT conditions can provide a valuable means for predicting their actual in vivo activity under natural or clinical conditions following consumption. The SLAP from lactobacilli have been reported to exhibit good tolerance to digestive enzymes and show lower degrees of degradation in the intestinal tract than that of the stomach (Xiao, 2012), findings that agree with our current results. Sun et al. (2012) reported that SLAP could protect lactobacilli cells against the hos-
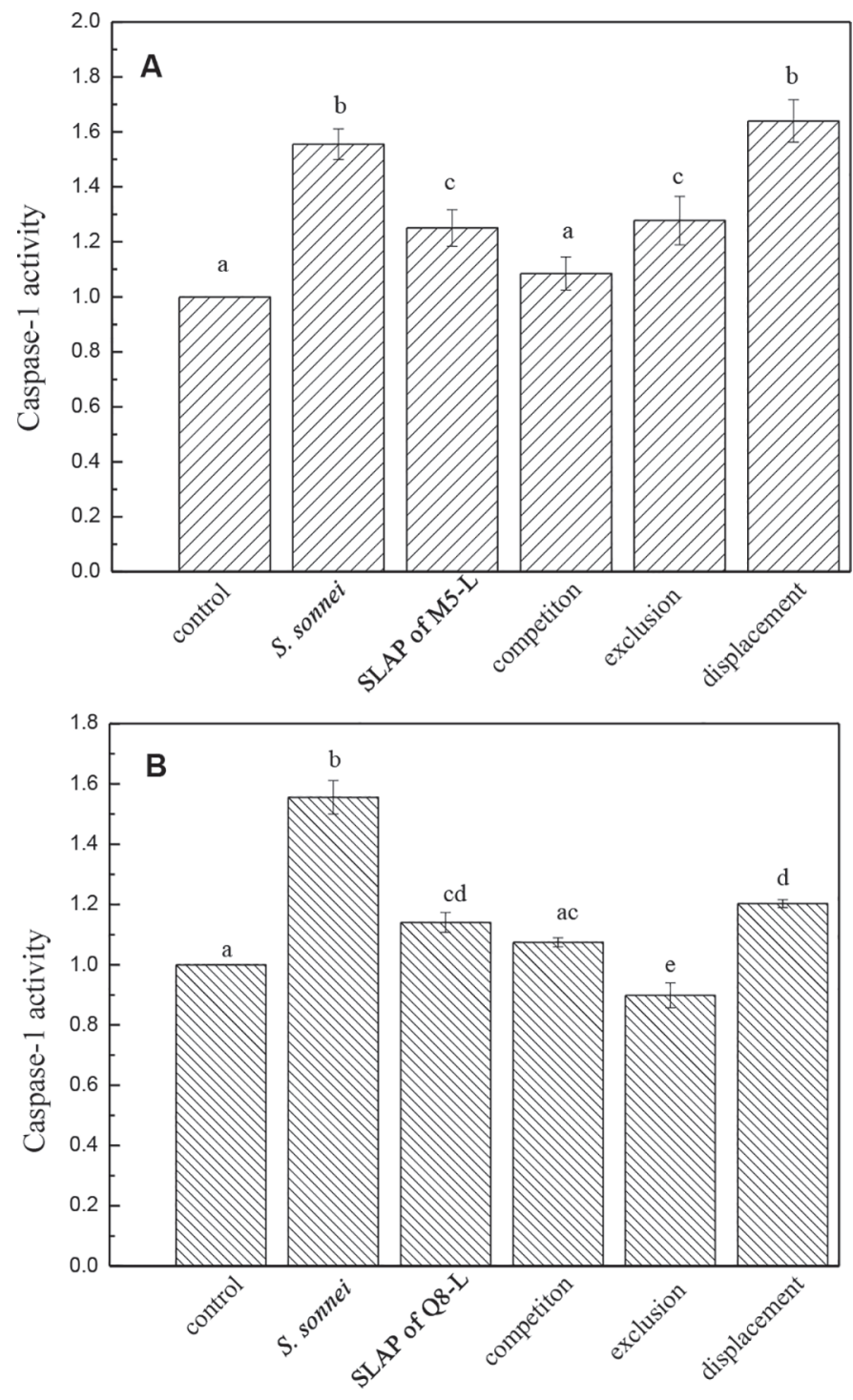

Figure 4. Function of surface-layer associated proteins (SLAP) of Lactobacillus paracasei ssp. paracasei M5-L (A) and Lactobacillus casei Q8-L (B) in caspase-1 activity assays. S. sonnei $=$ Shigella sonnei. Data are means $\pm \mathrm{SD}$ of 3 independent experiments. Letters $(\mathrm{a}-\mathrm{e})$ indicate differences between assays.

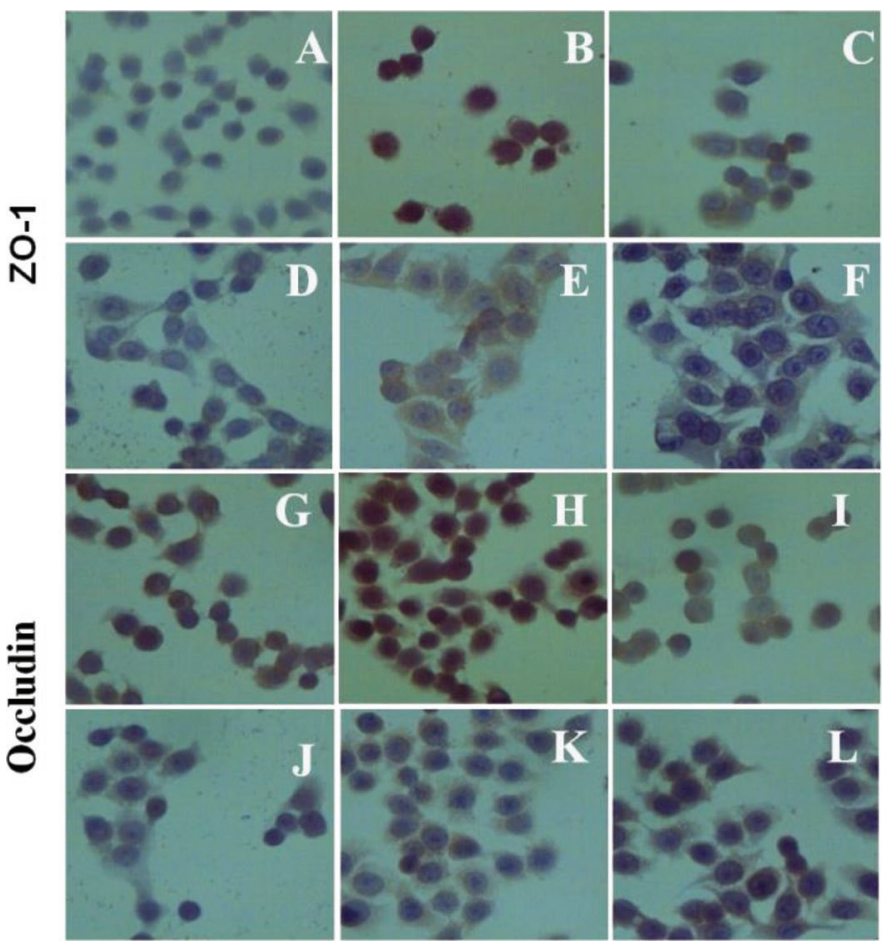

Figure 5. The surface-layer associated proteins (SLAP) of lactobacilli inhibited Shigella sonnei-induced ZO-1 and occludin redistribution. (A) Control group (uninfected HT-29 cells) for ZO-1; (B) treatment of HT-29 cells with SLAP of Lactobacillus paracasei ssp. paracasei M5-L alone; (C) treatment of HT-29 cells with SLAP of Lactobacillus casei Q8-L alone; (D) treatment of HT-29 cells with S. sonnei alone; (E) HT-29 cells infected with S. sonnei and co-incubated with SLAP of M5-L; (F) HT-29 cells infected with S. sonnei and coincubated with SLAP of Q8-L; (G) control group (uninfected HT-29 cells) for occludin; (H) treatment of HT-29 cells with SLAP of M5-L alone; (I) treatment of HT-29 cells with SLAP of Q8-L alone; (J) treatment of HT-29 cells with $S$. sonnei alone; (K) HT-29 cells infected with $S$. sonnei and co-incubated with SLAP of M5-L; and (L) HT-29 cells infected with $S$. sonnei and co-incubated with SLAP of Q8-L. Magnification: $200 \times$. Color version available online.

tile environment resulting from secretions of the small intestine. Our current results demonstrate that SLAP of M5-L were partially degraded under the simulated gastric conditions in our experiment. Accordingly, it would be necessary to use some type of microcapsule to protect SLAP of M5-L in the stomach to ensure their function within the intestinal tract. In contrast, the SLAP of Q8-L remained at $45 \mathrm{kDa}$ under these simulated conditions, which suggests that they would have the capacity for transport into the intestine through the stomach. Although the use of lactobacilli-induced SLAP against pathogen infection has been widely studied, the functional mechanisms underlying these effects have received much less attention. It has been proposed that these surface proteins may alter the competitive binding of intestinal epithelial cells to prevent bacterial adhesion and invasion; therefore, we directed our 
efforts at examining some of the potential mechanisms involved.

The findings of Xue et al. (2015) clearly showed that SLAP from $L$. paracasei M7 were responsible for their adherence to HT-29 cells. Moreover, they can inhibit the adhesion of Salmonella to HT-29 cells, as Salmonella was reduced by $42 \%$ in their exclusion assay experiment compared with that of controls. In our current experiments, we demonstrated that SLAP extracted from Q8-L and M5-L were ineffective in inhibiting the growth of S. sonnei, in agreement with results of $\mathrm{Li}$ et al. (2011). However, the SLAP of Q8-L and M5-L could directly inhibit $S$. sonnei adhesion to HT-29 cells as revealed in competition, exclusion, and displacement assays, although no obvious linear relationship was observed between inhibition rates and SLAP concentrations. Prophylactic use of SLAP may prove more effective than therapeutic use.

The SLAP of L. acidophilus ATCC 4356 can reduce apoptosis induced by Salmonella typhimurium SL1344, and this reduction in apoptosis is closely related to the time of application, with a maximal inhibitory effect observed at $36 \mathrm{~h}$ (Li et al., 2011). During pathogeninduced apoptosis, some critical factors are activated, such as caspase-1. Caspase-1 is a key executioner caspase in the proteolytic cascade that leads to apoptotic cell death, and it cleaves several structural proteins during the execution phase of apoptosis (Müller et al., 2009). In our experiment, we showed that $S$. sonnei was capable of invading epithelial cells, leading to cell apoptosis. Of greater significance were the findings that SLAP of Q8-L and M5-L in HT-29 cells inhibited this $S$. sonnei-induced apoptosis, a process that, in part, involves the capacity for SLAP to inhibit caspase-1 activation.

Resta-Lenert and Barrett (2003) reported that both Streptococcus thermophilus and L. acidophilus increased the resistance of HT-29 and Caco-2 cells to membrane barrier disruption caused by the pathogen, while maintaining or increasing the phosphorylation of TJ proteins such as actinin and occludin. In those studies, the culture media and heat-inactivated bacteria did not change cell membrane resistance. Similarly, Lactobacillus rhamnosus and Bifidobacterium increased phosphorylation of ZO-1 and occludin and increased epithelial resistance (Mathias et al., 2010). These increases in resistance are accompanied by a decrease in claudin-2 protein expression and an increase in ZO-1 and occludin protein production. Such results suggest some of the basis for their effects in reducing intestinal cell permeability and improving disease-induced colitis. Our current results are closely related to these findings. Specifically, we show that the SLAP of M5-L and Q8-L not only provide partial protection of intestinal epithelial cells but also promote the production of ZO-1 and occludin in these cells.

\section{CONCLUSIONS}

Our results provide new insights into the function of SLAP of Q8-L and M5-L, which can inhibit the adherence of $S$. sonnei, as revealed by exclusion, competition, and displacement assays. We demonstrated that SLAP of Q8-L and M5-L may be directly involved in this process by reducing downstream caspase-1 activation, thus modulating apoptotic cell damage. In addition, these SLAP can partly protect intestinal epithelial cells and promote the production of ZO-1 and occludin in cells. Uncovering the mechanism involved could enable novel approaches for the use of SLAP in antagonizing S. sonnei infection.

\section{ACKNOWLEDGMENTS}

This work was financially supported by National Natural Science Foundation of China (31301515), Natural Science Foundation of Heilongjiang Province (C201433), China Postdoctoral Science Foundation (2012M510093, 2013T60382), and the Fundamental Research Funds for the Central Universities (Grant no. HIT.NSRIF.2012082; Harbin Institute of Technology).

\section{REFERENCES}

Allen, S., J. Sotos, M. J. Sylte, and C. J. Czuprynski. 2001. Use of Hoechst 33342 staining to detect apoptotic changes in bovine mononuclear phagocytes infected with Mycobacterium avium ssp. paratuberculosis. Clin. Diagn. Lab. Immunol. 8:460-464.

Anderson, M., P. J. Sansonetti, and B. S. Marteyn. 2016. Shigella diversity and changing landscape: Insights for the twenty-first century. Front. Cell. Infect. Microbiol. 6:45.

Avall-Jääskeläinen, S., A. Lindholm, and A. Palva. 2003. Surface display of the receptor-binding region of the Lactobacillus brevis $\mathrm{S}$ layer protein in Lactococcus lactis provides nonadhesive lactococci with the ability to adhere to intestinal epithelial cells. Appl. Environ. Microbiol. 69:2230-2236.

Beveridge, T. J., P. H. Pouwels, M. Sára, A. Kotiranta, K. Lounatmaa, K. Kari, E. Kerosuo, M. Haapasalo, E. M. Egelseer, I. Schocher, U. B. Sleytr, L. Morelli, M. L. Callegari, J. F. Nomellini, W. H. Bingle, J. Smit, E. Leibovitz, M. Lemaire, I. Miras, S. Salamitous, P. Béguin, H. Ohayon, P. Gounon, M. Matuschek, and S. F. Koval., 1997. Functions of S-layers. FEMS Microbiol. Rev. 20:99-149.

Breuer, U. 2009. Lactobacillus molecular biology. From genomics to probiotics. Biotechnol. J. 4:769.

Bron, P. A., P. van Baarlen, and M. Kleerebezem. 2011. Emerging molecular insights into the interaction between probiotics and the host intestinal mucosa. Nat. Rev. Microbiol. 10:66-78.

Candela, M., F. Perna, P. Carnevali, B. Vitali, R. Ciati, and P. Gionchetti. 2008. Interaction of probiotic Lactobacillus and Bifidobacterium strains with human intestinal epithelial cells: Adhesion properties, competition against enteropathogens and modulation of IL-8 production. Int. J. Food Microbiol. 125:286-292.

Charteris, W. P., P. M. Kelly, L. Morelli, and J. K. Collins. 1998. Development and application of an in vitro methodology to determine the transit tolerance of potentially probiotic Lactobacillus and Bi- 
fidobacterium species in the upper human gastrointestinal tract. J. Appl. Microbiol. 84:759-768.

Chen, X. Y., J. J. Xu, J. B. Shuai, J. S. Chen, Z. F. Zhang, and W. H. Fang. 2007. The S-layer proteins of Lactobacillus crispatus strain ZJ001 is responsible for competitive exclusion against Escherichia coli O157:H7 and Salmonella typhimurium. Int. J. Food Microbiol. 115:307-312.

Golowczyc, M. A., P. Mobili, G. L. Garrote, A. G. Abraham, and G. L. De Antoni. 2007. Protective action of Lactobacillus kefir carrying S-layer protein against Salmonella enterica serovar Enteritidis. Int. J. Food Microbiol. 118:264-273.

Granato, D., F. Perotti, I. Masserey, M. Rouvet, M. Golliard, A. Servin, and D. Brassart. 1999. Cell surface-associated lipoteichoic acid acts as an adhesion factor for attachment of Lactobacillus johnsonii La1 to human enterocyte-like Caco-2 cells. Appl. Environ. Microbiol. 65:1071-1077.

Jakava-Viljanen, M., and A. Palva. 2007. Isolation of surface (S) layer protein carrying Lactobacillus species from porcine intestine and faeces and characterization of their adhesion properties to different host tissues. Vet. Microbiol. 124:264-273.

Khang, Y. H., H. Y. Park, Y. S. Jeong, J. A. Kim, and Y. H. Kim. 2009. Recombinant S-layer proteins of Lactobacillus brevis mediating antibody adhesion to calf intestine alleviated neonatal diarrhea syndrome. J. Microbiol. Biotechnol. 19:511-519.

Li, P. C., X. L. Ye, and Q. Yang. 2012. Antagonistic activity of Lactobacillus acidophilus ATCC 4356 S-layer protein on Salmonella enterica ssp. enterica serovar Typhimurium in Caco-2 cells. Ann. Microbiol. 62:905-909.

Li, P. C., Y. Y. Yin, Q. H. Yu, and Q. Yang. 2011. Lactobacillus acidophilus S-layer protein-mediated inhibition of Salmonella-induced apoptosis in Caco-2 cells. Biochem. Biophys. Res. Commun. 409:142-147

Lortal, S., J. V. Heijenoort, K. Gruber. And, and U. B. Sleytr. 1992 S-layer of Lactobacillus helveticus ATCC 12046: Isolation, chemical characterization and re-formation after extraction with lithium chloride. J. Gen. Microbiol. 138:611-618.

Lu, R. Y., W. X. Yang, and Y. J. Hu. 2014. The role of epithelial tight junctions involved in pathogen infections. Mol. Biol. Rep. 41:6591-6610

Maragkoudakis, P. A., C. Miaris, P. Rojez, N. Manalis, F. Magkanari, G. Kalantzopoulos, and E. Tsakalidou. 2006. Production of traditional Greek yoghurt using Lactobacillus strains with probiotic potential as starter adjuncts. Int. Dairy J. 16:52-60.

Mathias, A., M. Duc, L. Favre, J. Benyacoub, S. Blum, and B. Corthésy. 2010. Potentiation of polarized intestinal Caco-2 cell responsiveness to probiotics complexed with secretory IgA. J. Biol. Chem. 285:33906-33913.

Müller, A. J., C. Hoffmann, M. Galle, A. V. D. Broeke, M. Heikenwalder, L. Falter, B. Misselwitz, M. Kremer, R. Beyaert, and W. D. Hardt. 2009. The S.typhimurium effector SopE induces caspase-1 activation in stromal cells to initiate gut inflammation. Cell Host Microbe 6:125-136.

Ramiah, K., C. A. van Reenen, and L. M. T. Dicks. 2008. Surfacebound proteins of Lactobacillus plantarum 423 that contribute to adhesion of Caco-2 cells and their role in competitive exclusion and displacement of Clostridium sporogenes and Enterococcus faecalis. Res. Microbiol. 159:470-475.

Resta-Lenert, S., and K. E. Barrett. 2003. Live probiotics protect intestinal epithelial cells from the effects of infection with enteroinvasive Escherichia coli (EIEC). Gut 52:988-997.
Riedl, S. J., and G. S. Salvesen. 2007. The apoptosome: Signalling platform of cell death. Nat. Rev. Mol. Cell Biol. 8:405-413.

Servin, A. L. 2004. Antagonistic activities of lactobacilli and bifidobacteria against microbial pathogens. FEMS Microbiol. Rev. 28:405-440.

Singh, R., S. Pervin, G. Y. Wu, and G. Chaudhuri. 2001. Activation of caspase-3 activity and apoptosis in MDA-MB-468 cells by Nw-hydroxy-L-arginine, an inhibitor of arginase, is not solely dependent on reduction in intracellular polyamines. Carcinogenesis 22:1863-1869

Sleytr, U. B. 1976. Self-assembly of the hexagonally and tetragonally arranged subunits of bacterial surface layers and their reattachment to cell walls. J. Ultrastruct. Res. 55:360-377.

Sleytr, U. B., B. Schuster, E. M. Egelseer, and D. Pum. 2014. S-layers Principles and applications. FEMS Microbiol. Rev. 38:823-864.

Smit, E., F. Oling, R. Demel, B. Martinez, and P. H. Pouwels. 2001. The S-layer protein of Lactobacillus acidophilus ATCC 4356: Identification and characterisation of domains responsible for S-protein assembly and cell wall binding. J. Mol. Biol. 305:245-257.

Sun, Z. L., L. H. Huang, J. Kong, S. M. Hu, X. W. Zhang, and W. B. Kong. 2012. In vitro evaluation of Lactobacillus crispatus K313 and K243: high-adhesion activity and anti-inflammatory effect on Salmonella braenderup infected intestinal epithelial cell. Vet. Microbiol. 159:212-220.

Sun, Z. L., J. Kong, S. M. Hu, and W. T. Kong. 2013. Characterization of a S-layer protein from Lactobacillus crispatus K313 and the domains responsible for binding to cell wall and adherence to collagen. Appl. Microbiol. Biotechnol. 97:1941-1952.

Šušković, J., B. Kos, J. Beganovic', A. L. Pavunc, K. Habjanič, and S. Matošic'. 2010. Antimicrobial activity-the most important property of probiotic and starter lactic acid bacteria. Food Technol. Biotechnol. 48:296-307.

Tran Van Nhieu, G., R. Bourdet-Sicard, G. Duménil, A. Blocker, and P. J. Sansonetti. 2000. Bacterial signals and cell responses during Shigella entry into epithelial cells. Cell. Microbiol. 2:187-193.

Xiao, R. 2012. Screening of the Lactobacillus brevis M8 and cloning and expression analysis of its surface layer gene slpM. PhD Diss. Hunan Agricultural University, Hunan, China.

Xue, C. H., L. W. Zhang, R. B. Fan, S. M. Wang, H. B. Li, X. Luo, W. L. Liu, and W. Song. 2015. Salmonella infection and mediated inhibition of Salmonella-induced apoptosis. Eur. Food Res. Technol. 240:1-7.

Zhang, W. M., H. F. Wang, J. X. Liu, Y. H. Zhao, K. Gao, and J. Zhang. 2013. Adhesive ability means inhibition activities for lactobacillus against pathogens and S-layer protein plays an important role in adhesion. Anaerobe 22:97-103.

Zhang, Y. C., X. L. Xiang, Q. H. Lu, L. W. Zhang, F. Ma, and L. Wang. 2016. Adhesions of extracellular surface-layer associated proteins in Lactobacillus M5-L and Q8-L. J. Dairy Sci. 99:10111018.

Zhang, Y. C., L. W. Zhang, D. Ming, H. X. Yi, C. Guo, and Y. F. Tuo. 2011. Antimicrobial activity against Shigella sonnei and probiotic properties of wild lactobacilli from fermented food. Microbiol. Res. $167: 27-31$.

Zhang, Y. C., L. W. Zhang, Y. F. Tuo, C. F. Guo, H. X. Yi, J. Y. Li, X. Han, and M. Du. 2010. Inhibition of Shigella sonnei adherence to HT-29 cells by lactobacilli from Chinese fermented food and preliminary characterization of S-layer protein involvement. Res. Microbiol. 161:667-672. 